\title{
Freqüência e atividade enzimática de Candida spp. na cavidade oral de pacientes diabéticos do serviço de endocrinologia de um hospital de Fortaleza-CE
}

\author{
Frequency and enzymatic activity of Candida spp. oral cavity of diabetic patients of the service of \\ endocrinology of a hospital of Fortaleza-CE
}

Everardo Albuquerque Menezes'; Kristopherson Lustosa Augusto²; Caio César Furtado Freire²; Francisco Afrânio Cunha³; Renan Magalhães Montenegro4; Renan Magalhães Montenegro Júnior ${ }^{5}$

unitermos
Candida spp.
Diabetes mellitus
Cavidade oral
Atividade enzimática

resumo

O diabetes mellitus (DM), uma doença endócrino-metabólica de alta e crescente prevalência, é citada como responsável pela ocorrência das candidíases orais. A candidíase constitui um espectro de infecções causadas por fungos do gênero Candida, sendo o seu agente mais comum a Candida albicans, embora outras espécies tenham sido identificadas (Candida tropicalis, Candida guillermondii, Candida glabrata, Candida krusei). O objetivo deste trabalho foi avaliar a freqüência e a atividade enzimática de Candida spp. na cavidade oral de pacientes diabéticos atendidos no Serviço de Endocrinologia do Hospital Universitário Walter Cantídio da Universidade Federal do Ceará (HUWC/UFC). Foram coletadas amostras de 48 pacientes diabéticos, de ambos os sexos, com situações variáveis de controle glicêmico. Os materiais clínicos foram colhidos com ajuda de swabs e semeados em placas de Petri contendo ágarSabouraud dextrose com cloranfenicol e incubado a $37^{\circ} \mathrm{C}$. Os crescimentos foram identificados pelas provas clássicas usadas em micologia. Depois, essas cepas de Candida foram submetidas a provas de detecção de enzimas fosfolipase e proteinase. Destas, 15 amostras $(31,25 \%)$ apresentaram cultura positiva para o gênero Candida. A espécie mais freqüente foi a $C$. albicans, com $80 \%$, seguida de $C$. tropicalis (13,3\%) e C. guillermondii (6,7\%). Quanto à pesquisa da atividade enzimática de Candida spp., foi observado que $86,6 \%$ delas apresentaram atividade de proteinase e $80 \%$, de fosfolipase. Conclui-se com tais resultados que a $C$. albicans é a mais freqüente e que as espécies de Candida isoladas possuem fortes atividades enzimáticas.

\section{abstract}

Diabetes mellitus, a endocrine-metabolic disease, of high and increasing prevalence, is cited as responsible by the occurrence of oral candidiasis. Candidiasis constitutes a specter of infections caused by fungi of genera Candida; the most common agent is Candida albicans, but other species have also been identified (Candida tropicalis, Candida guillermondii, Candida glabrata, Candida krusei). The objective of this work was to evaluate frequency and enzymatic activity of Candida spp. in the oral cavity of diabetic patients taken care in the service of endocrinology of the University Hospital Walter Cantídio of the Federal University of the Ceará. Samples had been collected of 48 diabetic patients, men and women, with various situations of glicemic control. Clinical materials had been collected with aid of swab and harvested in plates of Petri contend Sabouraud agar dextrose with cloranfenicol and incubated to $37^{\circ} \mathrm{C}$. The grown were identified by the used classic tests in mycology. In the following, these Candida strains were submitted to tests to detect phospholipase and proteinase enzymes. Of these, 15 samples $(31,35 \%)$ presented positive culture for the genera Candida. The species more frequent was C. albicans with $80 \%$, followed by C. tropicalis (13.3\%) and C. guilliermondii (6.7\%). Asfor the research on the enzymatic activity of Candida sp. it was observed that $86.6 \%$ presented activity of proteinase and $80 \%$ of phospholipase. It was concluded with these results that $\mathrm{C}$. albicans is more frequent and that Candida spp. isolated species have strong enzymatic activity. key words

Candida spp.

Diabetes mellitus

Oral cavity

Enzymatic activity

1. Professor-doutor do Departamento de Análises Clínicas e Toxicológicas da Universidade Federal do Ceará (DACT/FFOE/UFC).

2. Médicos.

3. Farmacêutico do DACT/FFOE/UFC.

4. Professor-doutor; chefe do Serviço de Endocrinologia do Hospital Universitário Walter Cantídio (HUWC) da UFC.

5. Professor-doutor; coordenador do Serviço de Endocrinologia do HUWC/UFC. 


\section{Introdução}

A candidíase constitui um espectro de infecções causadas por fungos do gênero Candida. $\mathrm{O}$ agente mais comum é a Candida albicans, mas outras espécies também têm sido identificadas, como, por exemplo, C. tropicalis, C. guillermondii, C. glabrata, C. krusei, entre outras ${ }^{(2)}$.

Vários fatores são citados como responsáveis pela ocorrência das candidíases orais, alguns relacionados ao hospedeiro e outros à própria levedura. Os mais citados relacionados ao hospedeiro são: diabetes mellitus (DM), perda de dentes, restaurações, aparelhos (ortodônticos e protéticos), mudanças de hábitos alimentares, higiene oral, doenças sistêmicas, alterações hormonais, certas drogas sistêmicas e locais, imunodepressão, radiação e quimioterapia ${ }^{(12)}$.

Entre os fatores relacionados com a levedura (também denominados fatores de virulência) encontram-se o fenômeno de aderência (adesina), a forma miceliana, a variabilidade fenotípica, a produção de enzimas extracelulares (protease e fosfolipase) e as toxinas. Após a adesão da levedura às células do hospedeiro, inicia-se a pseudofilamentação da mesma, já que é uma característica da vida parasitária ${ }^{(12,20)}$.

O DM tem sido considerado um fator predisponente para candidíase ${ }^{(15)}$. Nesse distúrbio ocorre uma diminuição da capacidade defensiva dos neutrófilos polimorfonucleares (PMN) e dos linfócitos T relacionada com a hiperglicemia, gerando um meio favorável para a reprodução de espécies de Candida ${ }^{(19)}$.

A patogenia das Candida spp. está relacionada a uma combinação de fatores que contribuem para a sua virulência, destacando-se a produção de enzimas extracelulares, como a proteinase e a fosfolipase, como um dos principais mecanismos de virulência. $\mathrm{O}$ amplo espectro da candidíase e a sua significância clínica têm estimulado o interesse no estudo desses mecanismos de patogênese $\mathrm{e}^{(1)}$.

É importante ressaltar que estas leveduras podem fazer parte da microbiota normal e ser encontradas facilmente como simples comensais. Portanto, o diagnóstico da candidíase depende do isolamento em cultura e da demonstração da patogenia da espécie isolada ${ }^{(5)}$.

Assim, o objetivo deste trabalho foi avaliar a freqüência e os principais fatores de virulência de Candida spp. na mucosa oral de pacientes diabéticos.

\section{Material e métodos}

Foram coletadas amostras de material da mucosa oral de 48 pacientes adultos diabéticos, homens e mulheres, com- pensados e descompensados, provenientes do Ambulatório de Endocrinologia do Hospital Universitário Walter Cantídio da Universidade Federal do Ceará (HUWC/UFC), no ano de 2002. Esses pacientes foram selecionados aleatoriamente durante consultas de acompanhamento ambulatorial. A coleta do material foi realizada usando-se dois swabs estéreis, sendo um para esfregaço em lâmina e coloração pela técnica de Gram e outro destinado ao cultivo ${ }^{(10,11)}$.

Os materiais clínicos obtidos através da coleta foram semeados com um swab pela técnica de esgotamento, em placas de Petri contendo ágar-Sabouraud dextrose com cloranfenicol e incubado a $37^{\circ} \mathrm{C}$. Os crescimentos foram identificados pelas seguintes provas: teste do tubo germinativo, microcultivo em ágar-fubá, teste para fermentação de açúcares (zimograma) e assimilação de açúcares (auxonograma) $^{(7,8)}$.

As espécies de Candida foram submetidas a provas de detecção de enzimas fosfolipase e proteinase. Suspensões das leveduras em água destilada estéril, equivalente a 1 da escala de MacFarland, foram inoculadas em pontos equidistantes, respectivamente, nos meios de ágar-proteinase (Difco) e ágar-fosfolipase (Difco). As placas contendo dois inóculos de diferentes cultivos permaneceram incubadas a $37^{\circ} \mathrm{C}$ durante sete dias para proteinase e quatro dias para fosfolipase $\mathrm{i}^{(14,16,21)}$.

A presença da enzima proteinase foi observada pela formação de um halo transparente ao redor da colônia da levedura, e a presença da fosfolipase, pela formação de uma zona opaca ao redor da colônia da levedura. As atividades enzimáticas (PZ) foram medidas dividindo-se o diâmetro da colônia pelo diâmetro da colônia mais a zona de precipitação. Em ambos os casos, quando o PZ foi 1, a atividade enzimática foi zero; e quando a medida do PZ ficou entre 0,64 e 0,99, sua atividade foi considerada positiva $^{(14,16,21)}$.

\section{Resultados}

Neste estudo foram analisadas 48 amostras de material colhido da mucosa oral dos pacientes diabéticos. Dessas, 15 (31,2\%) apresentaram cultura positiva para o gênero Candida.

A espécie mais freqüente foi a C. albicans, com $80 \%$, seguida de $C$. tropicalis $(13,3 \%)$ e C. guillermondii $(6,7 \%)$, de acordo com a Figura 1.

Quanto à pesquisa da atividade enzimática de Candida spp., foi observado que 13 apresentaram PZ entre 0,64 
e 0,99 , isto é, $86,6 \%$ das cepas produziram proteinase, sendo 12 de Candida albicans e uma de Candida tropicalis. Em relação à fosfolipase, 12 cepas apresentaram $\mathrm{PZ}$ entre 0,64 e 0,99 , isto é, $80 \%$ produziram fosfolipase (Figura 2), sendo todas essas cepas de Candida albicans. Em relação ao tubo germinativo observou-se que essas 12 cepas de Candida albicans apresentaram esse importante fator de virulência.

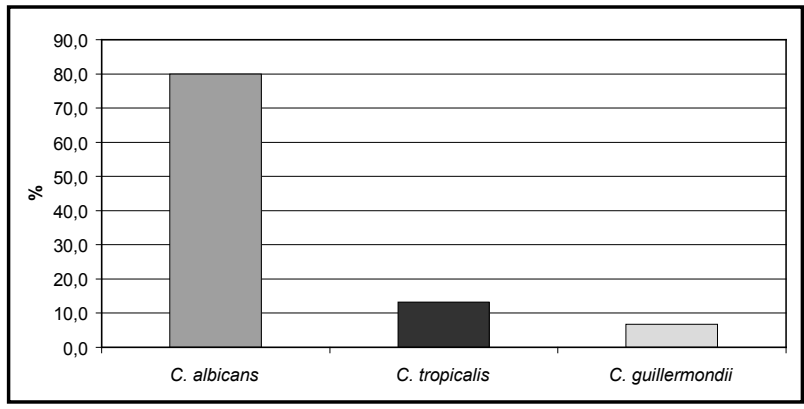

Figura 1 - Freqüência de espécies isoladas do gênero Candida spp. de amostras da mucosa oral dos pacientes diabéticos do Ambulatório de Endocrinologia do HUWC/UFC

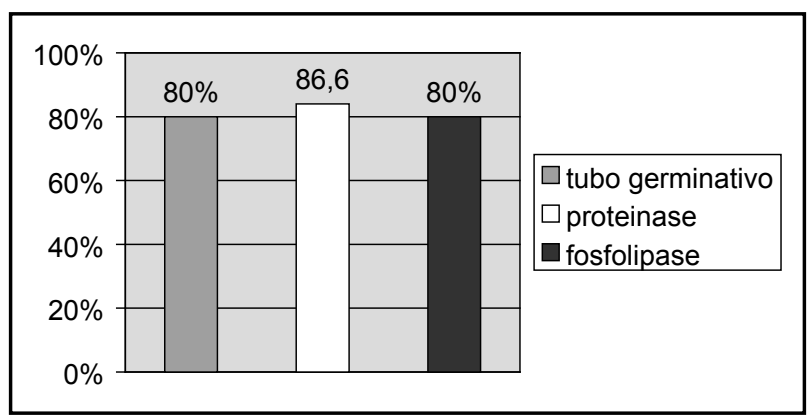

Figura 2 - Fatores de virulência em Candida spp. isoladas de pacientes diabéticos do Ambulatório de Endocrinologia do HUWC/UFC

\section{Discussão}

Vários estudos têm comprovado a suscetibilidade de pacientes diabéticos a infecções fúngicas e colonização da cavidade oral por espécies de Candida ${ }^{(12)}$. Além da colonização na cavidade oral, López-Martínez ${ }^{(9)}$ encontrou diferentes tipos de micoses (candidose, dermatofitose e otomicose) em $54,5 \%$ de 66 pacientes diabéticos. Hill et al.(6) observaram uma prevalência de $49 \%$ de candidose bucal em 51 diabéticos. No nosso estudo, verificamos uma porcentagem de $31,25 \%$ de candidose.

Segundo Fongsmut et al. ${ }^{(3)}, C$. albicans é a mais freqüente espécie de Candida isolada na cavidade oral de diabéticos e pacientes saudáveis, sendo que existe uma prevalência significativamente mais relevante em diabéticos. No entanto, como contraponto, há artigos científicos que contradizem essas afirmações.
No presente estudo a C. albicans foi a espécie de Candida mais comum entre os pacientes diabéticos analisados (Figura 1), corroborando o estudo de Fongsmut et al. ${ }^{(3)}$. Os autores, estudando a mucosa bucal de pacientes com diabetes, encontraram uma freqüência de $85 \%$ de Candida albicans, dados bastante semelhantes aos do presente estudo, onde foram encontrados $80 \%$ de Candida albicans.

Não foi observada uma relação clara entre os níveis de glicose e a colonização por Candida spp., já que 44,45\% dos pacientes estavam compensados; e foram isoladas Candida spp., dado que se contrapõe aos resultados de Gayosso e Mendez-Tovar ${ }^{(4)}$, em que todos os casos de infecção por C. albicans foram colhidos de pacientes descompensados.

No presente trabalho, a produção de proteinase foi altamente prevalente $(86,6 \%)$ nas amostras isoladas (Figura 2). Até o presente momento, nenhum estudo havia avaliado a atividade enzimática de Candida isolada de amostras da cavidade oral de pacientes com diabetes. Oliveira ${ }^{(11)}$ observou atividade fortemente proteolítica em pacientes imunocomprometidos, ressaltando a importância desta enzima no processo de patogênese. Sousa et al. ${ }^{(18)}$ verificaram que, em pacientes portadores de prótese total, as atividades enzimáticas de $C$. albicans foram de $52 \%$ para produção de proteinase. Candido et al.(1) observaram que $66,7 \%$ de Candida albicans isoladas na mucosa bucal de pacientes da Faculdade de Odontologia de Ribeirão Preto apresentaram atividade proteolítica. Penha et al.(13) verificaram, em pacientes da Faculdade de Odontologia da Universidade de São Paulo (USP), que $100 \%$ apresentaram atividade proteolítica, demonstrando forte positividade.

Em relação à produção de fosfolipase, os nossos resultados mostraram uma atividade fosfolipásica (das C. albicans isoladas) em $80 \%$ das C. albicans isoladas (Figura 2), semelhante aos trabalhos realizados por outros autores ${ }^{(1,10,11,13,18)}$. Candido et al..$^{(1)}$ e Penha et al. ${ }^{(13)}$ verificaram que as cepas de $C$. albicans isoladas da mucosa bucal apresentaram uma atividade fosfolipásica de 83,3\%.

Considerando as duas atividades, fosfolipásica e proteolítica, em $80 \%$ das cepas testadas, poderíamos sugerir uma correlação entre a produção dessas enzimas e sua patogenia, principalmente se isoladas de indivíduos considerados clinicamente sadios, para adotar métodos preventivos $^{(17) \text {. }}$

A presença de fatores de virulência nas amostras isoladas de pacientes diabéticos sugere a importância do estudo sistemático desses fatores, visto que a candidíase 
bucal pode ser o foco de disseminação da infecção nessa condição.

Estudos posteriores serão necessários para determinar o real impacto desses achados sobre a disseminação da infecção.

\section{Agradecimentos}

Os autores agradecem aos funcionários do Serviço de Endocrinologia do HUWC/UFC, pois sem eles este trabalho não poderia ter sido realizado.

\section{Referências}

1. CANDIDO, R. C.; AZEVEDO, R. V. P.; KOMESU, M. C. Enzimotipagem de espécies do gênero Candida isoladas da cavidade bucal. Rev Soc Bras Med Trop, v. 33, n. 5, p. 437-42, 2000.

2. CRISSEY, J.; LANG, H.; PARISH, L. C. Manual of medical mycology. Philadelphia: Blackwell Science, 1995. 263p.

3. FONGSMUT, T.; DEEROCHANAWONG,C.; PRACHYABRUED, W. Intraoral Candida in Thai diabetics patients. J Med Assoc Thai, v. 81, n. 6, p. 449-53, 1998.

4. GAYOSSO, P. M.; MÉNDEZ-TOVAR, L. V. Frecuencia de micosis superficiales en pacientes diabéticos de consulta externa. Derm Rev Mex, v. 39, n. 6, p. 33942. 1995.

5. GHANNOUM, M. A.; ABU-ELTENN, K. H. Pathogenicity determinants of Candida. Mycoses, v. 33, p. 265-82. 1990.

6. HILL, L. V. et al. Association of oral candidiasis with diabetic control. J Clin Pathol, v. 42, p. 502-5, 1989.

7. KREGER-VAN RIJ, N. J. W. The yeast: a taxonomy study. 3. ed. Amsterdam: Elsevier Science Publishers B. V., 1984.

8. LACAZ, C. S. et al. Tratado de micologia médica Lacaz. São Paulo: Editora Sarvier, 2002. 1104p.

9. LÓPEZ-MARTÍNEZ, R. Infección micótica en el paciente diabético. Rev Med IMSS, v. 13, p. 245-50, 1974.

10. MAFFEI, C. M. L. Amostras de Candida albicans isoladas de gestantes: fatores de virulência, sensibilidade a antifúngicos, tipagem fenotípica e genotípica. 1996. 183p. Tese (Doutorado) - Departamento de Microbiologia do Instituto de Ciências Biomédicas da Universidade de São Paulo, São Paulo.

11. OLIVEIRA, M. T. B. Estudo da mucosa bucal de pacientes imunocomprometidos no Estado do Rio Grande do Norte. 1993. 135p. Dissertação (Mestrado) - Departamento de Microbiologia do Instituto de Ciências Biomédicas da Universidade de São Paulo, São Paulo.
12. PAULA, C. R. Candidíases. In: ZAITZ, C. et al. Compêndio de micologia médica. Rio de Janeiro: MEDSI, 1998. p. 99-107.

13. PENHA, S. S. et al. Frequency and enzymatic activity (proteinase and phospholipase) of Candida albicans from edentulous patients, with and without denture stomatitis. Pesq Odont Bras,v. 14, n. 2, p. 119-22, abr/jun, 2000.

14. PRICE, M. F.; WILKINSON, I. D.; GENTRY, I. O. Plate method for detection of phospholipase activity in Candida albicans. Sabouraudia, v. 20, p. 15-20, 1982.

15. QUIRINO, M. R. S. et al. Distribution of oral yeasts in controlled and uncontrolled diabetic patients. Rev Microbiol, v. 25, n. 1, p. 37-41, 1994.

16. RÜCHEL, R.; TEGELER, R.; TROST, M. A comparison of secretory proteinases from different strains of Candida albicans. Sabouraudia, v. 20, p. 233-44, 1982.

17. SARMARANAYAKE, L. P.; RAESIDE, J. M.; McFARLANE, T. W. Factors affecting the phospholipase activity of Candida espécies in vitro. Sabouraudia, v. 22, p. 201-7, 1984.

18. SOUSA, P. C. P. et al. Fatores de virulência de Candida albicans isoladas de pacientes que fazem uso de prótese total na cidade de Fortaleza. Rev Bras Odontol, v. 60, n. 6, p. 390-3, 2003.

19. VARTITARIAN, S.; SMITH, C. B. Pathogenesis: host resistance and predisponing factors. In: BODEY, G. P. (ed.). Candidiasis. Pathogenesis, diagnosis and treatment. 2. ed. New York: Raven Press, 1993. p. 59-84, v. 4.

20. VIDOTTO, V. et al. Correlation between germ tube production, phospholipase activity and serotype distribution in Candida albicans. Rev Iberoam Mico., v. 16, p. 208-10, 1999

21. WILLIAMSON, M. I.; SAMARANAYAKE, L. P.; MAC FARLANE, T. W. Phospholipase activity as a criterion for biotyping Candida albicans. J Med Vet Myc, v. 24, p. 415-7, 1986. 